\title{
Identification and Antibiotic Susceptibility Testing of ESBL Producing Klebsiella Strains by Phenotypic Methods
}

\author{
Taqdees Malik*, Asma Naim* and Asma Saeed \\ Department of Microbiology, University of Karachi, Karachi, Pakistan
}

\section{A BSTRACT}

Multi drug resistance has now become a worldwide therapeutic challenge due to the widespread use of broad spectrum antibiotics. Klebsiella species have significant importance in clinical field as they cause various infections in human and are considered as potential pathogens that express antibiotic resistance through their strong enzymatic activity. Extended spectrum beta lactamases (ESBLs) are plasmid mediated enzymes produced mostly because of mutation and few other factors. These enzymes confer resistance against various $\beta$-lactam drugs including cephalosporins and monobactams. Among the genus Klebsiella, ESBLs are highly prevalent in $K$. pneumoniae followed by $K$. oxytoca.

This study was conducted in Pakistan to assess the distribution of ESBL producers among Klebsiella spp., an important member of the family Enterobacteriaceae. From January 2010 to January 2012, a total of 236 gram-negative isolates were collected from different renowned microbiological laboratories. Out of the 236 gram-negative isolates, 125 were found as Klebsiella spp. by using standard microbiological techniques. Antimicrobial susceptibility profiling of these strains was performed by using Kirby Bauer disk diffusion method. Phenotypic detection of the production of extended spectrum beta lactamase enzyme was performed using double disc synergy method and combination disc method.

It has been identified that Klebsiella strains are highly resistant against Amoxicillin, Tetracycline, Nalidixic Acid, Cephradine, Gentamicin, co-amoxyclav with the percentage of $100 \%, 86 \%, 86 \%, 82 \%, 82 \%$ and $80 \%$ respectively. The most effective antibiotics for Klebsiella spp. were found to be Amikacin, Meropenem and Piperacillin-tazobactam, with highest sensitivities of $96 \%, 94 \%$ and $91 \%$. Phenotypic detection of Extended spectrum beta lactamase production by double disc synergy test was able to identify $28 \%$ ESBL producers among Klebsiella isolates whereas $64 \%$ were detected by combination disc test.

Keywords:

ESBL, Klebsiella, DDST, CDT.
${ }^{*}$ Address of Correspondence:

anaeem@uok.edu.pk

taqdees_13@hotmail.com
Article info.

Received: June 21, 2018

Accepted: July 06, 2018

Cite this article: Malik T, Naim A, Saeed A. Identification and antibiotic susceptibility testing of ESBL producing Klebsiella strains by phenotypic methods. RADS J. Biol. Res. Appl. Sci. 2018; 9(1): 8-13.

This is an Open Access article distributed under the terms of the Creative Commons Attribution License (http://creativecommons.org/licenses/by/4.0), which permits unrestricted use, distribution, and reproduction in any medium, provided the original work is properly cited.

\section{INTRODUCTION}

Klebsiella is a well-known genus of the family Enterobacteriaceae associated with nosocomial and community-acquired pneumonia with high morbidity and mortality rates, if not treated timely and properly. Klebsiella as an opportunistic pathogen is mainly involved

\section{Funding Source: Nil} Conflict of Interest: Nil 
with human clinical specimens, accounting for $75 \%$ to $86 \%$ and $13 \%$ to $25 \%$ isolates respectively2.

The continuous spread of drug resistance among pathogenic microbes has become a therapeutic challenge all over the world. The indiscriminate and widespread use of extended spectrum antibiotics has played a significant role for the spread of multiple drug resistance among pathogenic strains ${ }^{3}$. Klebsiella isolates are gaining importance due to the emergence of their multidrug resistant strains, specifically extended-spectrum beta lactamase(ESBL)-producing strains, capable of hydrolyzing beta lactam drugs ${ }^{1}$. The first strain capable of producing ESBL was detected in Germany in 1983 and later several other outbreaks were reported in USA and other regions due to these ESBL producers ${ }^{4}$.

ESBLs are plasmid mediated enzymes that are capable of transferring resistance from one strain to another, not only for beta lactam drugs, they may also carry resistance genes of aminoglycosides, fluoroquinolones, tetracyclines, chloramphenicol and sulfamethoxazoletrimethoprim. These ESBLs can hydrolyze third generation cephalosporins including cefotaxime, ceftriaxone, ceftazidime and monobactams but could be inactivated by serine $\beta$-lactamase inhibitors such as clavulanate, sulbactam and tazobactam when used in combination with third generation cephalosporins ${ }^{3}$.

The timely and accurate detection of ESBL producers can prevent several outbreaks and endemics. Although various molecular techniques are now known for the detection of ESBL producers, however, in routine lab practice, they are rarely followed. It is therefore suggested to use two or more phenotypic detection methods for identification of ESBL producers in routine clinical settings to avoid misuse of antibiotics ${ }^{3}$.Prevalence, susceptibility profile and phenotypic characteristics of ESBL producers may vary from country to country and region to region. Therefore, in our study, we performed susceptibility testing and phenotypic detection of ESBL producer and ESBL non-producers among Klebsiella strains to establish effective antibiotic strategy and suggest appropriate empirical therapy in high-risk units.

The present study was carried out to determine the antibiotic resistance pattern and phenotypic and detection of resistance enzymes in Klebsiella species for implementing the strategies and judicial use of drugs for proper therapy. For executing strict infection control policy and to stop the spread of resistant strains, it is important to detect ESBL producing isolates more promptly.

\section{MATERIALS AND METHODS}

\section{Bacterial Isolates:}

From January 2010 to January 2012, a total of 236 gramnegative isolates were collected from renowned microbiological laboratories of Karachi, Pakistan. All the samples were aseptically streaked on Nutrient Agar slants (Oxoid). Identification and characterization of bacterial isolates was performed by using standard microbiological techniques ${ }^{7}$. Identified isolates were stored at $-70^{\circ} \mathrm{C}$ inGlycerol stock ${ }^{8}$.

\section{Antibiotic Susceptibility Testing:}

Antibiotic Susceptibility testing was performed by KirbyBauer method. Antimicrobial susceptibilities of the isolates to 25 different antimicrobial agents $(\mu \mathrm{g})$ viz. Amikacin (30), Gentamicin (10), Tobramycin (10), Amoxicillin (20), Amoxicillin/clavulanic acid (20/10), Piperacillintazobactam (100/10), Ciprofloxacin (5), Ofloxacin (5), Enoxacin (10), Sparfloxacin (5), Nalidixic Acid (30), Cephradine/ Velosef (30), Cefuroxime (30), Cefixime (5), Cefotaxime (30), Ceftazidime (30), Ceftriaxone (30), Cefoperazone/Sulbactam (75/30), Imipenem (10), Meropenem (10), Doxycycline (30), Tetracycline (30), Trimethoprim/sulphamethoxazole

Fosfomycin(200) and Nitrofurantoin (300) were determined by using commercially available disks (Oxoid) and results were interpretedas per National Committeefor Clinical Laboratory Standards (NCCLS) guidelines?.

\section{Phenotypic Detection of ESBL Producers:}

Extended spectrum beta lactamase producing strains among Klebsiella isolates were detected by the methods of double disc synergy and combination disc.

\section{Double Disc Synergy Test:}

The test was performed by using disks of Cefotaxime (30 ug) (Oxoid ${ }^{\mathrm{TM}}$ ) and Amoxicillin Clavulanic acid $(20 / 10 \mathrm{ug})\left(\right.$ Oxoid $\left.^{\mathrm{TM}}\right)$. Disc of Cefotaximewas placed at $15 \mathrm{~mm}$ from Amoxicillin Clavulanic aciddisc and incubated at $37^{\circ} \mathrm{C}$ for 24 hours. Synergy between the discs was observed and the increased zone of CTX towards AMC was 
considered as a clear indication for ESBL production ${ }^{10}$.

\section{Combination Disc Method:}

Cefotaxime (30ug) (Oxoid ${ }^{\mathrm{TM}}$ ) and the combined disc of Cefotaxime plus Clavulanic acid (30/10 ug) $\left(\right.$ Bioanalyse $^{\circledR}$ ) were used in this test following protocol recommended by CLSI in 2010. Both discs were placed at a distance of $24 \mathrm{~mm}$ to observe the zones of inhibition. ESBL producers were detected by comparing the zone sizes of the discs. ESBL production can be interpreted by $5 \mathrm{~mm}$ increase in the zone size of combination disc as compared to the cefotaxime disc ${ }^{9-11}$.

\section{RESULTS}

Out of 125 identified Klebsiella strains, 100 strains were isolated from female patients and 25 strains were from male patients. Klebsiella isolates were detected higher in the patients of younger age followed by the older group. Less number of isolates were detected in the middle-aged patientsand children. Distribution of Klebsiella isolates with respect to the age group has been shown in Fig. 1 .

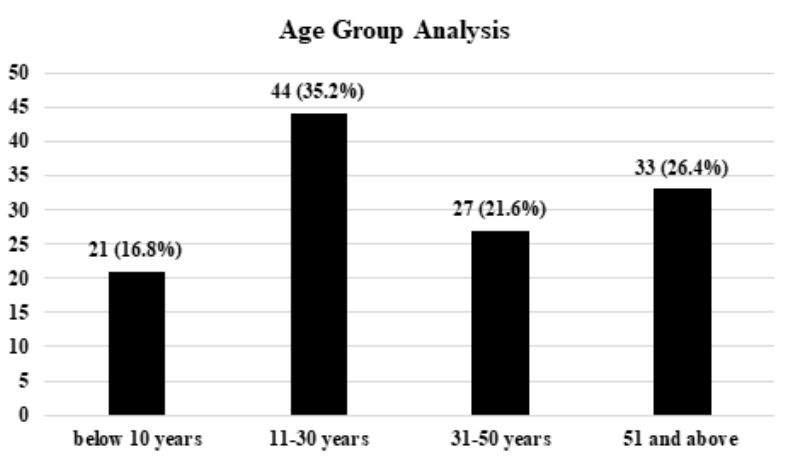

Fig. 1: Age group analysis showing the number of Klebsiella isolates collected from selected age groups.

Susceptibility testing of strains detected that amikacin, meropenem, imipenem and piperacillin-tazobactam are the most effective antimicrobial agents against Klebsiella isolates. It was found that only $8 \%$ isolates were resistant to Imipenem, $6 \%$ showed resistance against meropenem, $3 \%$ to Amikacin, and $2 \%$ to Piperacillin-tazobactam. We detected resistance against amoxicillin in $100 \%$ strains of Klebsiella as the isolates were intrinsically resistant to penicillin. Highest resistance was observed against Nalidixic acid (86\%), Doxycycline (86\%), Tetracycline
(86\%), Gentamicin \& Cephradine (82\%) and Amoxicillin/clavulanic acid (Co-Amoxyclav) (80\%).

Resistance pattern of Klebsiella strains against other antimicrobials was detected as $63 \%$ against Cefuroxime, $42 \%$ to Cefixime; $36 \%$ to Ciprofloxacin, $33 \%$ to Sparfloxacin, 30\% to Enoxacin, 29\% to Ofloxacin and Cefotaxime whereas $26 \%$ resistance was observed against Ceftazidime, Ceftriaxone and Cefoperazone/Sulbactam and $19 \%$ isolates were resistant to Tobramycin. Complete Resistance profile including number of sensitive, intermediate and resistant strains are mentioned in Fig. 2.

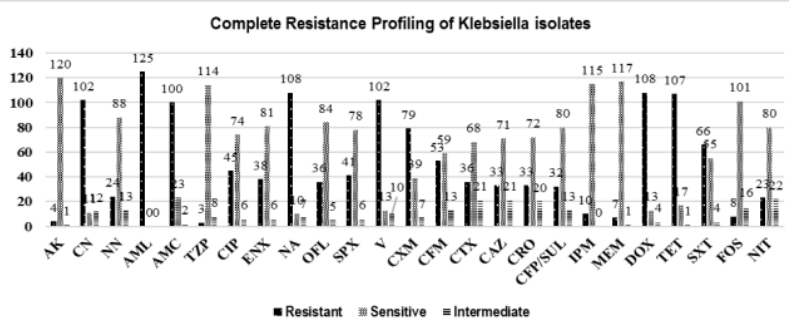

Fig. 2: Antibiotic resistance pattern of Klebsiella species against multiple drugs.

Out of 125 identified strains of Klebsiella, 35 were found as ESBL producers by Double disc synergy test whereas 80 strains were detected as ESBL producers by combination disc method as mentioned in Table 1. Detection of ESBL producers due to size and variation in zones of inhibitions for both methods has been illustrated in Fig. 3.

Table 1: Number and percentage of ESBL positive Klebsiella isolates using DDST and CDT.

\begin{tabular}{cc}
\hline Test & $\begin{array}{c}\text { No. of ESBL positive } \\
\text { isolates(n) Percentage (\%) }\end{array}$ \\
\hline Double Disc Synergy Test & $35(28)$ \\
\hline Combination Disc Test & $80(64)$ \\
\hline $\begin{array}{c}\text { Total No. of ESBL producers } \\
\text { by both method }\end{array}$ & $81(64.8)$ \\
\hline
\end{tabular}




\section{Double Disc Synergy Test}

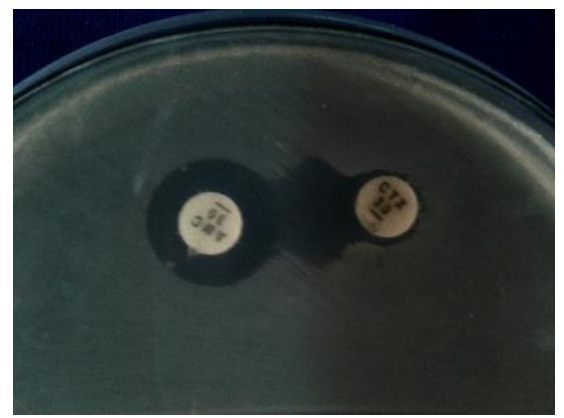

(A): Double Disc Synergy Test positive Klebsiella isolate showing keyhole appearance.

\section{Combination Disc Test}

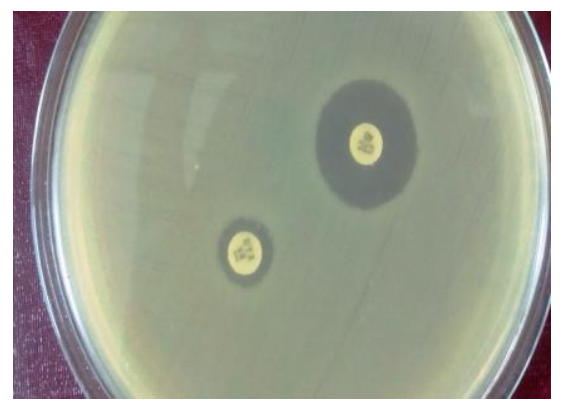

(B): Combination Disc Test positive Klebsiella isolate showing extended zone of inhibition in CTX+CA as compared to CTX alone.

Fig. 3: Comparison of (A) Double Disc Synergy Test and (B) Combination Disc Test.

\section{DISCUSSION}

Several reports and studies have been conducted on Klebsiella isolates, describing their occurrence, susceptibility pattern and ESBL production. Klebsiella strains have gained immense importance among the scientific community due to their multi drug resistance strains with a specific mechanism of resistance i.e. enzyme production. In our study we identified 125 Klebsiella strains with high occurrence among females and less in males. Our findings confirmed the studies reported from India, Nigeria, Iran in which Klebsiella spp. were detected higher in females as compared to males due to the compressed size of urethra,5,612-14. We detected that the prevalence of Klebsiella isolates was higher in the younger age group. Contradictory researches were reported from India and Iran in which higher number of isolates were detected in the middle-aged group and the age group of children below 2 years respectively.
Dissimilarity in the result can be occurred in different regions, especially it depends upon the consumption rate of antibiotics among different age groups ${ }^{5,14}$.

We determined the resistance profile of Klebsiella isolates by using twenty-five antimicrobial agents. We found that all Klebsiella isolates were found resistant to amoxicillin. Nalidixic Acid (NA), Doxycycline, Tetracycline, Gentamicin, Cephradine, and Amoxicillin/clavulanate were less effective antimicrobials against Klebsiella isolates. Moderate resistance was observed against cephalosporins and quinolones. However, carbapenems, amikacin and Piperacillin-tazobactam have shown good inhibitory results against Klebsiella strains. All strains of Klebsiella were resistant against amoxicillin. Few other studies reported $100 \%$ resistance against amoxicillin however, comparatively less resistance against coamoxiclav was reported in another research ${ }^{15}$. Moderate resistance to third generation cephalosporin and comparatively less resistance against piperacillin tazobactam and aminoglycosides ${ }^{16-18}$. Good susceptibility results were obtained with aminoglycosides and fluoroquinolones ${ }^{6}$.

In our study, we detected carbapenem as an effective drug against Klebsiella spp. However, contrary to our research, resistance against carbapenem drugs was also reported in Klebsiella isolates ${ }^{19}$. Although we did not obtain good inhibitory results from ciprofloxacin against Klebsiella isolates, however, few studies have reported ciprofloxacin as an effective drug against these strains ${ }^{16,20}$. A study reported that carbapenem should be considered as a drug of choice against the infections caused by Klebsiella isolates ${ }^{21}$. A similar study conducted in Australia, revealed that imipenem has the similar susceptibility pattern against ESBL producing isolates and ESBL non-producing isolates ${ }^{22}$. Klebsiella strains were found to be sensitive against amikacin and imipenem in the study ${ }^{23}$. Comparable results were reported in another study of Pakistan in which imipenem, piperacillintazobactam, ampicillin-sulbactam and amikacin were found as effective drugs ${ }^{24}$.

We detected $35(28 \%)$ and 80 (64\%) strains of ESBL producers by Double disc synergy test and by combination disc method respectively. ESBL producers were initially reported from Europe in 1983, since then their prevalence is increasing day by day. Various reports 
have revealed its prevalence in different regions of the world. ESBL production was detected in $30-60 \%$ isolates from Brazil in a study conducted on the patients of intensive care units ${ }^{21}$. In another study, $86 \%$ of Klebsiella strains were detected as ESBL producers by both methods ${ }^{17}$.

Combination Disc Method can be considered to be more reliable as it detects higher number of isolates in our research and this method was also verified by the fact that by adding clavulanic acid to third generation cephalosporin, it will increase its effectiveness. Similar phenomenon was stated in another research ${ }^{20}$. Comparatively lesser number (24.5\%) of ESBL producing strains were detected in Pakistan 201324. Another study from Pakistan reported 22\% ESBL producing strains among Klebsiella isolates in 201625. In 2016, a study reported fewer number of ESBL producers by using the method of Double disc synergy as compared to the combination disc method ${ }^{26}$. In a study conducted in Nepal, higher number of ESBL producing Klebsiella isolates $(18.4 \%)$ were reported by using the method of phenotypic confirmatory disc diffusion test ${ }^{27}$ whereas in 2017, ESBL production was reported in relatively less number of Klebsiella pneumoniae (3.8\%) and Klebsiella oxytoca $(0.7 \%)$ isolates ${ }^{28}$. Therefore, it would be preferable to conduct more than phenotypic screening technique to detect the correct number of ESBL producers.

\section{CONCLUSIONS}

This is an alarming situation that antibiotic resistance is increasing among pathogenic strains. Therefore, now there is an intense need to restrict the widespread use or misuse of antibiotics. Antibiotic susceptibility profiling and detection of ESBL producing strains must be included in the routine laboratory testing protocols. In the light of our findings, we could suggest that the use of amoxicillin and co-amoxiclav should be restricted. Cephalosporins could only be used in case of ESBL non-producers. However, Carbapenems, Amikacin and Piperacillin-tazobactam can be the drugs of choice for the treatment of infections caused by Klebsiella species. Phenotypic screening of ESBL producers can be better detected by the combination disc test or it is preferable to use more than one screening procedures for the detection of ESBL production.

\section{REFERENCES}

1. Podschun R, Ullmann U. Klebsiella spp. as nosocomial pathogens: epidemiology, taxonomy, typing methods, and pathogenicity factors. Clin Microbiol Rev. 1998; 11(4):589-603.

2. Pokra M, Sharma DK, Mehta P, Verma HR, Pundir S, Rana J, et al. Its Alarming, Klebsiella spp. towards Multidrug Resistance. Int J Curr Microbiol App Sci 2016; 5(6): 150-60.

3. Vinodhini R, Moorthy K, Palanivel P, Punitha T, Saranya $\mathrm{S}$, Bhuvaneshwari $\mathrm{M}$, et al. Detection and antimicrobial susceptibility pattern of ESBL producing Gram negative bacteria. Asian J Pharm Clin Res. 2014;7(1):243-7.

4. Behrooozi A, Rahbar M, Yousefi J.V. Frequency of extended spectrum beta-lactamase (ESBLs) producing Escherichia coli and klebseilla pneumoniae isolated from urine in an Iranian 1000-bed tertiary care hospital. Afr J Microbiol Res 2010; 4 (9): 881-8.

5. Akram M, Shahid M, Khan A.U. Etiology and antibiotic resistance patterns of community-acquired urinary tract infections in JNMC hospital Aligarh, India. Ann Clin Microbiol Antimicrob 2007; 23; 6: 4.

6. Chikwendu $\mathrm{Cl}$, Amadi ES, Obi RK. Prevalence and antimicrobial resistance in Pseudomonas aeruginosa and Klebsiella pneumoniae isolates from non-clinical urine samples. NY Sci J. 2010; 3(11): 194-200.

7. Krieg NR, Holt JC. (eds., 1984). Bergey's Manual of Systematic Bacteriology, 1st ed., vol. 1, Williams and Wilkins, Baltimore.

8. Cheesbrough M. 2006. A practical and well-illustrated guide to microbiological, hematological, and blood transfusion techniques. District Laboratory Practice in Tropical Countries - Part 2.

9. National Committee for Clinical Laboratory Standards (NCCLS) guidelines 2010.

10. Vaidya VK. Horizontal transfer of Antimicrobial Resistance by Extended-Spectrum $\beta$ LactamaseProducing Enterobacteriaceae. J Lab Physicians 2011; 3(1): 37-42.

11. Lin C-F, Hsu S-K, Chen C-H, Huang Jr-R, Lo H-H. Genotypic detection and molecular epidemiology of extended-spectrum $\beta$-lactamase-producing Escherichia coli and Klebsiella pneumoniae in a regional hospital in central Taiwan. J Med Microb 2010; 59: 665-71.

12. Khosravi $A D$, Hoveizavi $H$, Mehdinejad M. Prevalence of Klebsiella pneumoniae encoding genes for CTX-M-1, TEM-1 and SHV-1 extended-spectrum beta lactamases (ESBL) enzymes in clinical specimens. Jundishapur $\mathrm{J}$ Microbiol 2013; 6(10): e8256. 
13. Babakhani S, Derikvand SS, Nazer MR, Kazemi MJ. Comparison frequency and determination antibiotic resistance pattern of Klebsiella spp. Isolated from nosocomial infection in Khorramabad shohadayeashayer hospital. Bull Env Pharmacol Life Sci 2014; 3(12): 14954.

14. Akbar DA, Ali RA, Zahra KJ. Screening and prevalence of SHV/CTX-M/TEM $\beta$-lactamase resistance genes in Klebsiella strains isolated bacteria from urinary tract infection in pre-school-age children in Jahrom, Iran. IIOABJ 2016; 7 (10): 82-8.

15. Wagenlehner FME, Niemetz A, Dalhoff A, Naber KG. Spectrum and antibiotic resistance of uropathogens from hospitalized patients with urinary tract infections: 19942000. Int J Antimicrob Agen 2002; 19: 557-64.

16. Randrianirina $F$, Soares J-L, Carod J-F, Ratsima E, Thonnier V, Combe P, Grosjean P, Talarmin A. Antimicrobial resistance among uropathogens that cause community-acquired urinary tract infections in Antananarivo, Madagascar. J Antimicrob Chemother 2007; 59: 309-12.

17. Manchanda V, Singh NP, Goyal R, Kumar A, Thukral SS. Phenotypic characteristics of clinical isolates of Klebsiella pneumonia \& evaluation of available phenotypic techniques for detection of extended spectrum beta-lactamases. Indian J Med Res 2005;122: 330-7.

18. Kashef N, Djavid GE, Shahbazi S. Antimicrobial susceptibility patterns of community-acquired uropathogens in Tehran, Iran. J Infect Dev Ctries 2010; 4(4):202-6.

19. Bratu S, Tolaney P, Karumudi U, Quale J, Mooty $\mathrm{M}$, Nichani $\mathrm{S}$, et al. Carbapenemase-producing Klebsiella pneumoniae in Brooklyn, NY: molecular epidemiology and in vitro activity of polymyxin B and other agents. J Antimicrob Chemother 2005; 56: 128-32.

20. Colodner R. Extended-spectrum beta-lactamases: a challenge for clinical microbiologists and infection control specialists. Am J Infect Control 2005; 33(2):104-7.

21. Paterson DL, Bonomo RA. Extended spectrum $\beta$ lactamases: A clinical update. Clin Microbiol Rev. 2005; 18(4): 657-86.
22. Hawser SP, Bouchillon SK, Hoban DJ, Badal RE, Hsueh $P-R$, Paterson DL. Emergence of high levels of extended-spectrum-beta-lactamase-producing gramnegative bacilli in the Asia-Pacific Region: data from the study for monitoring antimicrobial resistance trends (SMART) Program, 2007. Antimicrob Agen Chemother 2009; p. 3280-4.

23. Abreu AG, Marques SR, Monteiro-Neto V, Gonçalves AG. Extended-spectrum beta-lactamase-producing enterobacteriaceae in community-acquired urinary tract infections in São Luís, Brazil. Braz J Microb 2013; 44(2): 469-71.

24. Ahmed I, Sajed M, Sultan A, Murtaza I, Yousaf $S$, Maqsood B, et al. The erratic antibiotic susceptibility patterns of bacterial pathogens causing urinary tract infections. EXCLI J 2015; 14: 916-25.

25. Ain NU, Abrar S, Nazeer K, Riaz S. Extended spectrum beta lactamases and metallobeta-lactamases: An update from Lahore, Pakistan Eur J Pharm Med Res 2016; 3(9): 92-7.

26. Yala J-F, Mabika RM, Bisseye C, Kenguele HM, Kama EM, Dikoumba AC, et al. Phenotypic and genotypic characterization of extended-spectrum-beta-lactamases producing-Enterobacteriaceae (ESBLE) in patients attending Omar Bongo Ondimba military hospital at Libreville (Gabon). J Mol Microbiol Biotechnol. 2016; 4(6): 944-9.

27. Chaudhary P, Bhandari D, Thapa K, Thapa P, Shrestha D, Chaudhary HK, Shrestha A, Parajuli H, Gupta BP. Prevalence of Extended Spectrum Beta-Lactamase Producing Klebsiella Pneumoniae Isolated from Urinary Tract Infected Patients. J Nepal Health Res Counc. 2016;14(33): 111-5.

28. Shakya P, Shrestha D, Maharjan E, Sharma VK, Paudyal R. ESBL Production Among E. coli and Klebsiella spp. Causing Urinary Tract Infection: A Hospital Based Study. Open Microbiol J. 2017; 11: 2330. 